\title{
DIGITAL TECHNOLOGIES IN PROVIDING DEVELOPMENT OF ALGORITHMS SURGICAL TREATMENT OF SUPRAVENTRICULAR ARRHYTHMIAS
}

\author{
Vladimir Evtushenko ${ }^{1,3 a}$, Konstantin Smyshlyaev ${ }^{3}$, Alexander Bykov $^{1}$, Yury Kistenev ${ }^{1}$, \\ Elena Pavlyukova ${ }^{3}$, Vadim Katkov ${ }^{3}$, Igor Kurlov ${ }^{3}$, Yana Anfinogenova ${ }^{2,3}$ and \\ Alexey Evtushenko ${ }^{1,3}$ \\ ${ }^{1}$ National Research Tomsk State University, 634050, Tomsk, Russia \\ ${ }^{2}$ National Research Tomsk Polytechnic University, 634050, Tomsk, Russia \\ ${ }^{3}$ Federal State Budgetary Scientific Institution "Research Institute for Cardiology", 634012, Tomsk, \\ Russia
}

\begin{abstract}
The aim of the study was the development and clinical application of patient selection algorithm for surgical treatment of longlasting persistent atrial fibrillation. The study included 235 patients with acquired heart disease and coronary artery disease, which in the period from 1999 to 2015 performed surgical treatment of long-term persistent atrial fibrillation (RF "MAZE III" procedure) in conjunction with the correction of the underlying heart disease. The patients were divided into 2 groups according to the method of operation: the group $1-135$ patients (76 women and 59 men) who have applied an integrated approach to surgery for atrial fibrillation, including penetrating method of RF effects on atrial myocardium and the study of the function of the sinus node before and after the operation (these patients were operated on from 2008 to 2015). The group $2-100$ patients (62 women and 38 men) with a "classical" method of monopolar RF "MAZE III", which the sinus node function was not studied. We used the combined (epi- and endocardial) method of RF «MAZE». This algorithm is decreasing of possible permanent pacemaker postoperatively. The initial sinus node function in these patients, measured using the original method, the basic line of this algorithm was taken. The results showed that use this algorithm for selection of patients allows significantly reduce the possibility of pacemaker implantation in the postoperative period.
\end{abstract}

\section{Introduction}

Improving the efficiency of radiofrequency (RF) ablation "MAZE III" due to sinus rhythm (grade 4 on a scale of Santa Crus) [1] is an actual problem in modern surgical arrhythmology.

\footnotetext{
${ }^{a}$ Corresponding author: evtushenko.vladimir@gmail.com
} 
The drug treatment of AF is usually ineffective and reduced to the control of ventricular rate and prevention of thromboembolic complications. Measures aimed at the preservation of sinus rhythm are effective only in paroxysmal AF when the main mechanism of its development is a trigger. When myocardial substrate for maintenance of AF is forming, and the micro re-entry is developing, drug therapy becomes ineffective [2-4]. Thus, the development of surgical treatment of persistent and prolonged persistent AF becomes especially over other methods.

At the same time, the effectiveness of carefully performed radiofrequency procedure "MAZE III" in some cases significantly reduced due to postoperative sinus node dysfunction. Therefore, even a well-executed surgery does not lead to a significant improvement in patient well-being. In addition, these patients are in need of long-term temporary pacing and prolonged presence in the intensive care unit, and in some cases they need the pacemaker implantation.

In a number of scientific papers was shown [3-8], that the long persistent AF reduces the likelihood of recovery of sinus rhythm after the procedure, "MAZE III", however, does not provide a method to estimate the initial functional state of the sinus node during AF. Atrial fibrillation makes excessive load on atrial cardiomyocytes, during episodes of atrial fibrillation. This leads to the activation of neurohumoral mechanisms (mainly cardiac angiotensin II), which trigger mechanism "structural remodeling", leading to dilatation, hypertrophy, structural disorganization of myofibrils fibrosis and atrial myocardium, that escalate the processes of "electrical remodeling" and supports AF [4, 9, 10]. Thus, atrial fibrillation itself maintains and exacerbates the atrial fibrillation, leading to cell degeneration of sinoatrial node.

Thus, the preservation of the sinoatrial node function in patients during atrial radiofrequency fragmentation scheme "MAZE III" is one of the leading problems.

In the literature, forecasting methods the recovery of sinoatrial node function after surgical treatment of AF with cardiopulmonary bypass has not been found. The close similar is a method for predicting the successful restoration of sinus rhythm after the procedure, "MAZE III", associated with the preoperative analysis of electrocardiograms and standard chest leads $[3,11,12]$. The method is a f-wave analysis on the electrocardiogram. Restoration of normal sinus rhythm is possible if the amplitude of the $f$ waves of more than $0.1 \mathrm{mV}$.

The method disadvantage is it does not provide direct information about the preoperative electrophysiological condition of sinoatrial node. This one allows to predict only the restoration of normal atrial contractile activity. It cannot give a reliable forecast for the restoration of normal sinoatrial node function after the procedure, "MAZE III". In addition, an important disadvantage is the fact that there is no clear algorithm for the selection of patients for atrial radiofrequency fragmentation scheme "MAZE III", is based on the prevention of sinoatrial node dysfunction.

The aim of study is prognosis normal sinus rhythm recovery RF procedure "MAZE III", performed with cardiopulmonary bypass.

\section{Materials and methods}

The study included 235 patients with acquired heart disease and coronary artery disease, which in the period from 1999 to 2016 performed surgical treatment of long-term persistent atrial fibrillation (RF "MAZE III" procedure) in conjunction with the correction of the underlying heart disease. The patients were divided into 2 groups according to the method of influence: the first (primary) group -135 patients ( 76 women and 59 men) who have applied an integrated approach to surgery for atrial fibrillation, including penetrating method of RF effects on atrial myocardium and the study of the function of the sinus node 
before and after the surgery (these patients were operated on from 2008 to 2015). The second group - 100 patients (62 women and 38 men) with a "classical" method of monopolar RF "MAZE III", which the sinus node function was not studied (operated from 1999 to 2008.) [13]. We used the combined (epi- and endocardial) method of RF «MAZE» in both groups. Clinical characteristics and operations by groups are shown in Table (Table 1).

All patients before surgery were classified as II-III functional class (FC) according to the classification of New-York Heart Association (NYHA). Heart diseases were diagnosed on the basis of anamnesis, results of physical and instrumental studies. All patients underwent preoperative echocardioscopy with the definition of the morphology of valvular lesions and the degree of hemodynamic transvalvular blood flow (gradient, valve orifice area, effective area and regurgitant volume, the ratio of the effective area of the valve opening in the patient's body surface area). The etiology of heart diseases and hemodynamic types listed in the table (Table 1). Both groups of patients are identical, the differences between them in terms of preoperative statistically insignificant. Beta-blockers received 221 patients (94\%), angiotensin converting enzyme (ACE) inhibitors and diuretics obtained all patients (Table 2). Some patients received prehospital digoxin and amiodarone. 5 days prior to surgery patients were canceled beta-blockers, amiodarone and digoxin. According to the test with a 6-minute walk 221 patient was classified to III functional class (FC) of heart failure (according to NYHA), 14 patients - to the class II of NYHA. Also, all patients recorded 12-lead ECG, according to testimony - Daily Holter ECG monitoring. All patients older than 40 years (men) and 45 years (women) before surgery performed coronary angiography. Sampling solid, inclusion criteria for patients were the presence long persistent atrial fibrillation, resistance to drug therapy, combined heart defects requiring surgical correction. Exclusion criteria were the presence of severe comorbidities, multiple organ dysfunction syndrome, intolerance of iodine preparations, patient reluctance to participate in the study.

In the first group, all patients underwent electrophysiological study (EPS) of the sinoatrial node function by proposed technology. We used diagnostic complex "Elcart-M" ("Electropulse", Tomsk). Electrophysiological study protocol included a portion of the right atrium stimulation in order to assess the automatic function of the sinoatrial node (the definition of recovery time of sinoatrial node function and correct recovery time of sinoatrial node function) and becomes overdrive stimulation of the right atrium to the development of AV block grade 2 ("Wenckebach point"). Electrophysiological study of preoperative sinoatrial node function performed intraoperatively before the heart structure separation and beginning of cardiopulmonary bypass. This was done to minimize the external impact on the sinoatrial node tissue. The study was carried out as follows: intraoperative transesophageal performed ultrasound of the heart and in the absence of thrombosis of heart cavities cardioversion was performed. In the case of sinus rhythm after 10 minutes was performed electrophysiological study of the function of the sinoatrial node. According to the results of preoperative EPS Group 1 patients were divided into 2 subgroups: Subroup 1 with normal sinus automatism (118 patients); Subroup 2 with signs of sinoatrial node dysfunction (17 patients). After the main phase of the operation and remove the cannula lung machine from the cavities of the heart and great vessels, in case of restoration of sinus rhythm, control performed EPS. 
Table 1. Clinical characteristics of patients.

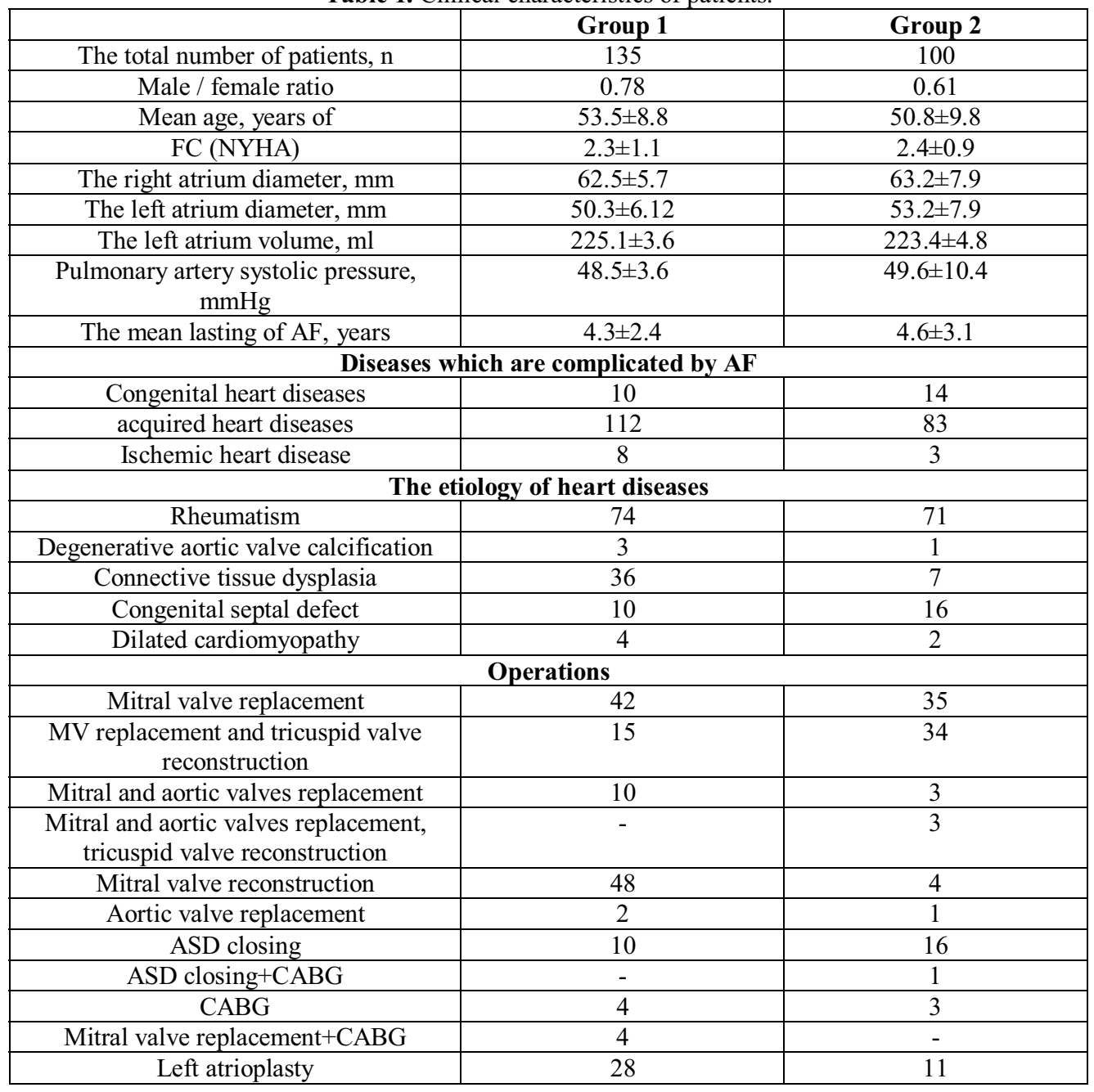

Statistical processing of the data was performed using the software package SPSS 15.0 for Windows (SPSS Software Products). Compliance testing samples of normal distribution was performed Shapiro-Wilk test criterion (Shapiro-Wilk). Description of data was carried out using mean and standard deviation $-\mathrm{M} \pm \mathrm{StD}$. The Student's t-test was used for assess the reliability of inter-group differences. Paired Student's t-test was used for assess the reliability of intragroup differences (dynamics of indicators pre-surgery within one group). The significance level " $\mathrm{p}$ " was taken to be 0.05 , respectively, the confidence level $(\mathrm{p})=0.95$.

Table 2. Drug therapy before surgery.

\begin{tabular}{|c|c|c|}
\hline The group of drugs & Group 1 (n, \%) & Group 2 (n, \%) \\
\hline Beta-blockers & $129(90,6 \%)$ & $92(92 \%)$ \\
\hline ACE inhibitors & $135(100 \%)$ & $99(99 \%)$ \\
\hline Diuretics & $135(100 \%)$ & $100(100 \%)$ \\
\hline Digoxin & $22(16 \%)$ & $31(31 \%)$ \\
\hline Amiodarone & $42(31 \%)$ & $12(12 \%)$ \\
\hline
\end{tabular}




\section{Results}

As a result of the study, patients in Group 1 in the Subgroup 1 mean recovery time of sinoatrial node function and correct recovery time of sinoatrial node function initially was $1033.7 \pm 101.2 \mathrm{~ms}$ and $242.3 \pm 40.1 \mathrm{~ms}$ respectively. All patients of Subgroup $1 \mathrm{had}$ postoperative sufficient rate sinus rhythm. During the EPS after the intervention, the mean recovery time of sinoatrial node function and correct recovery time of sinoatrial node function in this subgroup was $1020.3 \pm 203.1 \mathrm{~ms}$ and $305 \pm 60.4 \mathrm{~ms}$, respectively (Figure 1, 2).

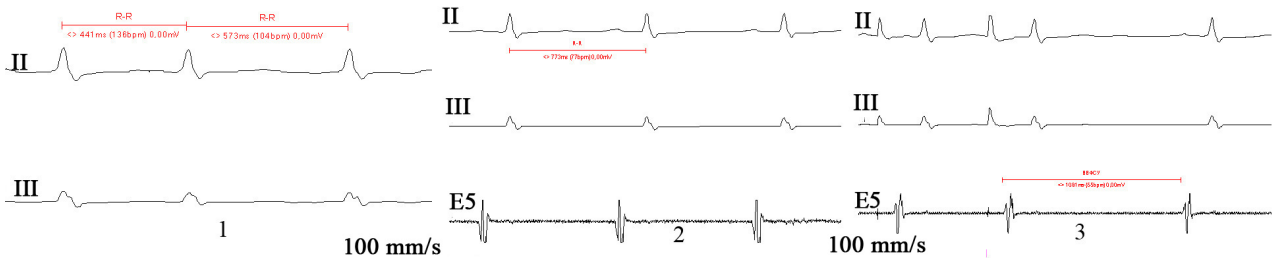

Figure 1. Patient's electrograms (subgroup 1) before surgery:

1) the original electrogram; I, II, III - standard leads; 2) the electrogram after cardioversion and sinus rhythm; I, II, III - standard leads; E5 - the sinoatrial node electrogram; 3) frequent stimulation of the right atrium in order to assess the automatic function of the sinoatrial node (recovery time of sinoatrial node function); I, II, III - standard leads; E5 - the sinoatrial node electrogram.
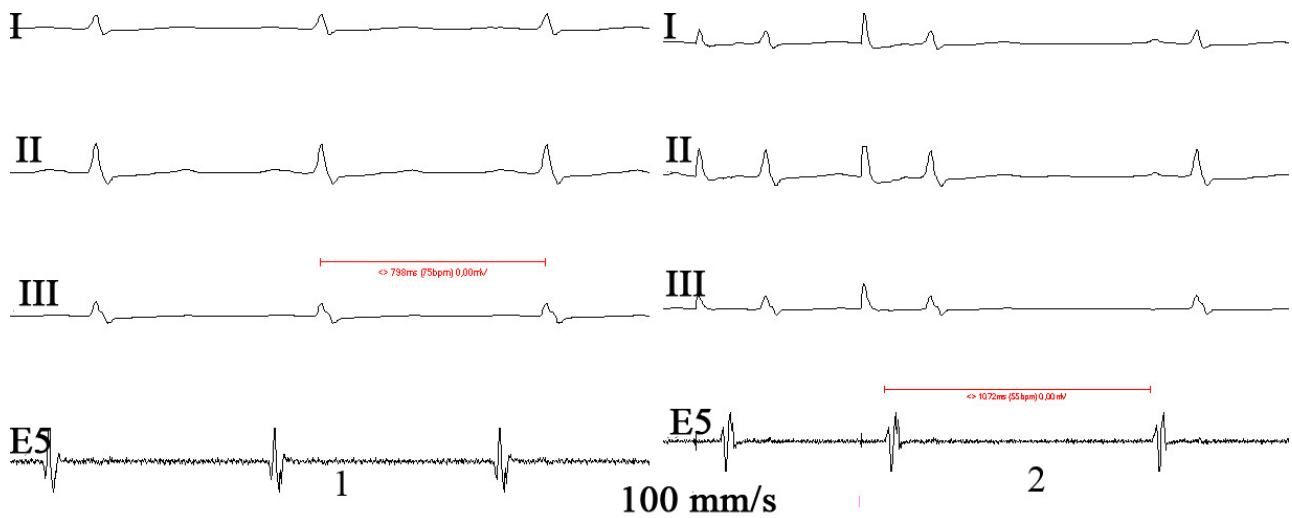

Figure 2. Patient's electrograms (subgroup 1) before surgery:

1)the original electrogram; I, II, III - standard leads; 2) frequent stimulation of the right atrium in order to assess the automatic function of the sinoatrial node (recovery time of sinoatrial node function); I, II, III - standard leads; E5 - the sinoatrial node electrogram.

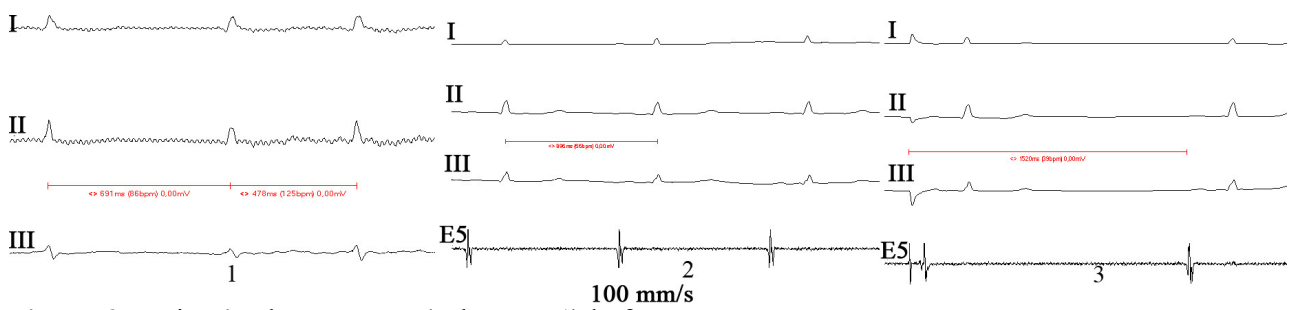

Figure 3. Patient's electrograms (subgroup 1) before surgery:

1) the original electrogram; I, II, III - standard leads; 2) the electrogram after cardioversion and sinus rhythm; I, II, III - standard leads; E5 - the sinoatrial node electrogram; 3) requent stimulation of the right atrium in order to assess the automatic function of the sinoatrial node (recovery time of sinoatrial node function); I, II, III - standard leads; E5 - the sinoatrial node electrogram. 
The recovery time of sinoatrial node function and correct recovery time of sinoatrial node function in patients of subgroup 2 initially had mean of $1995 \pm 30.1 \mathrm{~ms}$ and $971.5 \pm$ 102.4 ms, respectively (Figure 3).

In this subgroup of patients after surgery was observed sinoatrial node dysfunction and required temporary pacing mode AAI in up to 14 days (Figure 4). In 12 (71\%) patients in the Subgroup 2 for the early postoperative period AF returned. $5(29 \%)$ patients in this subgroup had pacemaker implantation. Thus, the total number of patients with irreversible sinoatrial node dysfunction was 17 people ( $12 \%$ of the patients of the group) $(0-1$ grading scale Santa Crus [1]).

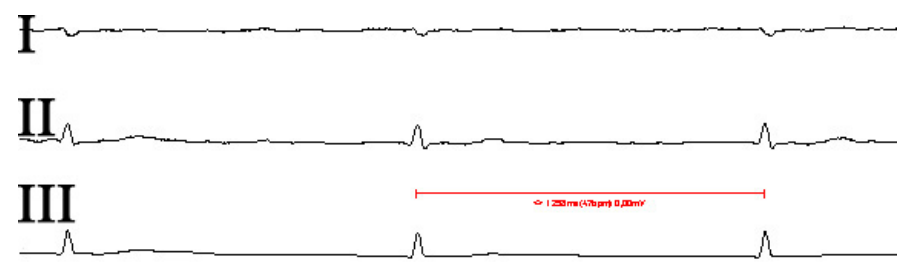

E5

\section{$100 \mathrm{~mm} / \mathrm{s}$}

Figure 4. The electrogram of the patient from subgroup 2 after surgery; I, II, III - standard leads; E5 - the sinoatrial node electrogram (no activity).

Patients of Subgroup 1 did not require a temporary pacemaker, on average, more than $3.4 \pm 2.1$ days. All of them had sinus rhythm sufficient rate.

Patients in group 2 had a significantly higher percentage of ineffective treatments "MAZE III", which consists in returning the AF and the need for pacemaker implantation $(\mathrm{p}<0.05)$ (Table 3).

Table 3. Early and long-term results by groups.

\begin{tabular}{|c|c|c|c|}
\hline \multirow{2}{*}{} & \multicolumn{2}{|c|}{$\begin{array}{c}\text { Group 1 } \\
\text { (N= 135) }\end{array}$} & \multirow{2}{*}{$\begin{array}{c}\text { Group 2 } \\
\text { (N=100) }\end{array}$} \\
\cline { 2 - 3 } & Subgroup 1 & Subgroup 2 & \\
\hline $\begin{array}{c}\text { Sinoatrial node dysfunction } \\
\text { (in terms of up to } 3.4 \pm 2.1 \\
\text { days) }\end{array}$ & $\begin{array}{c}56(47 \%) \\
(41 \% \text { of the total } \\
\text { number of patients } \\
\text { in the group) }\end{array}$ & $\begin{array}{c}17(100 \%)(12 \% \text { of the } \\
\text { total number of } \\
\text { patients in the group) }\end{array}$ & $86(86 \%)$ \\
\hline $\begin{array}{c}\text { Sinoatrial node dysfunction } \\
\text { (in terms of }>14 \text { days) }\end{array}$ & 0 & $\begin{array}{c}13(76 \%) \\
(9.6 \% \text { of the total } \\
\text { number of patients in } \\
\text { the group) }\end{array}$ & \\
\hline ECMO & \multicolumn{2}{|c|}{$3(2.2 \%)$} & $22 \%)$ \\
\hline Mortality & $2(1.5 \%)$ & $2(2 \%)$ \\
\hline Sinus rhythm in discharge & \multicolumn{2}{|c|}{$125(93 \%)$} & $78(78 \%)$ \\
\hline \multicolumn{2}{|c|}{ Long-terms results } \\
\hline
\end{tabular}




\begin{tabular}{|c|c|c|}
\hline $\begin{array}{c}\text { A) Sinus rhythm without } \\
\text { antiarrhythmic drugs }\end{array}$ & $120(88 \%)$ & $51(51 \%)$ \\
\hline $\begin{array}{c}\text { B) Sinus rhythm with } \\
\text { antiarrhythmic drugs }\end{array}$ & $126(93 \%)$ & $68(68 \%)$ \\
\hline Pacemaker & $5(3.7 \%)$ & $10(10 \%)$ \\
\hline Mortality & No & $\begin{array}{c}5(5 \%)(1 \text { patient } \\
\text { died of } \\
\text { hemorrhagic } \\
\text { stroke) }\end{array}$ \\
\hline $\begin{array}{c}\text { Thromboembolic } \\
\text { complications }\end{array}$ & $3.2 \%$ & \begin{tabular}{c} 
No \\
\hline Non-effective
\end{tabular} \\
\hline
\end{tabular}

In all patients, treatment of AF which has been found to be effective, there remains an active systole left and right atrium (A wave amplitude greater than $0.8 \mathrm{~m} / \mathrm{s}$ ). The A-wave is either absent or its amplitude $<0.5 \mathrm{~m} / \mathrm{s}$ in patients with sinoatrial node dysfunction.

\section{Discussion of the results}

Development of sinoatrial node dysfunction in patients with long-standing persistent atrial fibrillation is one of the reasons for the ineffectiveness of surgical treatment. This complication is not life-threatening in itself, but requires a long-term presence of the patient in intensive care, time spent pacing for a long time, and in some cases, the implantation of a permanent pacemaker [8, 14]. The sinoatrial node dysfunction develops as a result described by many researchers expressed the defeat of the host tissue and arteries, and its supply perinodal structures [5, 6, 15-17]. As pointed out by a number of researchers, sinoatrial node dysfunction provoked chronic AF, which in most cases is in these patients lengthening the time intraatrial and shortening of atrial refractoriness [3].

Possible causes of the sinoatrial node dysfunction after radiofrequency "MAZE" to "open" the heart are:

1. The mechanical damage of the sinoatrial node arteries during access to the mitral valve.

2. The thermal damage to the sinoatrial node during radiofrequency exposure on the myocardium.

3. The initial sinoatrial node dysfunction.

The first reason can be solved choice the atriotomy to the mitral valve $[18,19]$. When selecting the atriotomy approach to the mitral valve is necessary to consider the type of myocardial perfusion. In all cases, preferred the left atriotomy approach is not leading to damage of the arteries supplying the sinus node, but it is in some cases (at small sizes of left atria) provides visualization of mitral valve worse than the Guiraudon approach. When there is the right or balanced types of heart circulation, superior approach by Guiraudon is preferred. The transseptal approach to the mitral valve through right atria leads to damage of the arteries feeding the components of the conduction system in $100 \%$ cases [19].

To prevent thermal and wave damage to the sinus node is proposed and applied in clinic technique penetrating effects on atrial myocardium. Features of this method lies in the fact that the original design of the monopolar electrode allows for more effective impact on the transmural atrial myocardium in a shorter period of time, which reduces the area of damage and prevents involvement in the zone of influence elements of the conduction system of the heart [20]. 
However, to evaluate the functional state of the sinus before surgery during the phase transition was not possible. The literature found messages on technology research sinoatrial node functions in patients with long-term persistent AF. To solve this problem, we developed an original method of intraoperative evaluation sinoatrial node functions in these patients. It allows electrophysiological study with the definition of recovery time of sinoatrial node, correct the recovery time of sinus node function and "Wenckebach point" (becoming more frequent stimulation of the right atrium to the development of AV block degree 2).

Evaluation of sinoatrial node function using the technology developed before the procedure "MAZE III", correlates with its function in the late postoperative periods. Thus, the proposed technology allows to evaluate the function of the sinus node and conduction system of the heart in patients with long-standing persistent AF.

The findings suggest that while initially the compromised function of the sinoatrial node should not be expected in the postoperative effects of surgical treatment of AF in these patients. Moreover, given the initial severity of the condition of these patients, in some cases is unreasonable increase in the time of cardiopulmonary bypass to prevent complications, such as haemolysis, air embolism and systemic inflammatory response. Thus, to determine the indications for the procedure of "MAZE III" expedient to EPS on technology developed before the intervention with the assessment of the functional state of the sinoatrial node. When it is appropriate we need to assess the initial severity of the patient's condition and the effect on it of atrial fibrillation [21-25]. If AF has no pronounced negative effect on the hemodynamics of the patient, it makes sense to restrict the atrial appendages off by their resection and suturing, in addition to the main stage of the operation to reduce the risk of thromboembolic complications.

Thus, based on the above-described method for evaluating the function of sinus node selection algorithm can provide patients with long persistent AF for surgical treatment.

\section{Conclusion}

The proposed method for evaluating sinus node function in patients with long-standing persistent atrial fibrillation can be used to predict the successful restoration of sinus rhythm after the procedure, "MAZE III". A set of activities aimed at preserving the function of the sinus node, is able to increase the effectiveness of surgical treatment of atrial fibrillation. If there is the original sinus node dysfunction in patients, the surgical treatment of long-term persistent $\mathrm{AF}$ is not very promising in terms of the sinus rhythm restoration.

\section{Acknowledgements}

The study was supported by a grant from the Russian Science Foundation (№ 15-1510016), and the program of improving competitiveness of the TSU (project No. 8.2.31.2015).

\section{References}

[1] J. Q. Melo, J. Neves, P. Adragao, R. Ribeiras, M. M. Ferreira, L. Bruges, M. Canada, T. Ramos, European Journal of Cardio-Thoracic Surgery 12, 739 (1997) doi: 10.1016/S1010-7940(97)00252-2

[2] S. Benussi, A. Galanti, V. Zerbi, Y. A. Privitera, I. Iafelice and O. Alfieri, J. Thorac. Cardiovasc. Surg. 139, 1131 (2010) doi: 10.1016/j.jtcvs.2009.12.039 
[3] H.-Y. Chang, Y.-J. Lin, L.-W. Lo, S.-L. Chang, Y.-F. Hu, C.-H. Li, T.-F. Chao, W.H. Yin and S.-A. Chen, Europace 15, 205 (2013) doi: 10.1093/europace/eus219

[4] S. Fareh, C. Villemaire and S. Nattel, Circulation 98, 2202 (1998)

[5] A. Elvan, K. Wylie and D. P. Zipes, Circulation 94(11), 2953 (1996)

[6] J. Gomes and P. Kang, Circulation, 63, 80 (1981)

[7] J. M. S. Lee and J. M. Kalman, Europace 15, 161 (2013) doi: 10.1093/europace/eus223

[8] G. Peretto, A. Durante, L.R. Limite and D. Cianflone, Cardiology Research and Practice 2014, 615987 (2014) doi: 10.1155/2014/615987

[9] S. Saksena, A. Prakash, R. B. Krol and A. Shankar, Am. J. Cardiol. 84, 880 (1999) doi: 10.1016/S0002-9149(99)00459-2

[10] A. W. Teh, P. M. Kistler, G. Lee, C. Medi, P. M. Heck, S. J. Spence, P. B. Sparks, J. B. Morton and J. M. Kalman, J. Cardiovasc Electrophysiol 23, 232 (2012)

[11] A. S. Nemkov and A. V. Sydorchuk, Bulletin of Surgery 168, 21 (2009)

[12] H. G. Je, J. W. Lee, S. H. Jung, S. J. Choo, H. Song, S. C. Yun and C. H. Chung, European journal of cardio-thoracic surgery 36, $272 \quad$ (2009) doi: $10.1016 /$ j.ejcts.2009.02.058

[13] A. V. Evtushenko, M. B. Knyazev, V. M. Shipulin, S. V. Popov, I. V. Antonchenko, V. O. Kiselev, I. V. Ponomarenko, V. V. Evtushenko and M. V. Krivov, Siberian Medical Journal 2, 79 (2005)

[14] M. Pasic, M. Musci, H. Siniawski, O. Grauhan, B. Edelmann, T. Tedoriya, Y. Weng and R. Hetzer, J. Thorac. Cardiovasc. Surg. 118, 287 (1999) doi: 10.1016/S0022-5223(99)70219-9

[15] S. Saksena, I. Giorgberidze, R. Mehra, M. Hill, A. Prakash, R. B Krol and P. Mathew, Am. J. Cardiol. 83, 187 (1999) doi: 10.1016/S0002-9149(98)00822-4

[16] Y. H. Yeh, B. Burstein, X. Y. Qi, M. Sakabe, D. Chartier, P. Comtois, Z. Wang, C. T. Kuo and S. Nattel, Circulation 119, 1576 (2009)

[17] S. Nath and D. E. Haines, Prog. Cardiovasc. Dis. 37(4), 185 (1995) doi: 10.1016/S0033-0620(05)80006-4

[18] A. V. Evtushenko, V. V. Evtushenko, K. A. Petlin, E. M. Belenkova and I. V. Antonchenko, Herald of arrhythmology 69, 5 (2012)

[19] V. V. Evtushenko, A. V. Evtushenko, K. A. Petlin, V. M. Gulyaev, E. M. Belenkova and V. M. Shipulin, Clinical Physiology of circulation 4, 39 (2008)

[20] A. V. Evtushenko, V. V. Evtushenko, K. A. Petlin, E. M. Belenkova, E. K. Knyazeva, I. V. Antonchenko, V. Kh. Vaizov, V. M. Shipulin and S. V. Popov, Herald of arrhythmology, 48, 15 (2007)

[21] D. K. Swanson, W. J. Smith, T. Ibrahim and A. S. Wechsler, Innovations: Technology and Techniques in Cardiothoracic and Vascular Surgery 6(4), 276 (2011) doi: 10.1097/IMI.0b013e31822b4d22

[22] M. La Meir, S. Gelsomino, F. Lucà, R. Lorussoc, G. F. Gensinic, L. Pisond, F. Wellensb and J. Maessena, Interact. Cardiovasc. Thorac. Surg. 14, 445 (2012) doi: 10.1093/icvts/ivr142

[23] W. Saliba and O. M. Wazni, Clin Cardiol. 34, 12 (2011) doi: 10.1002/clc.20826

[24] C. Wenning, P. S. Lange, C. Schülke, A. Vrachimis, G. Mönnig, O. Schober, L. Eckardt and M. Schäfers, EJNMMI Res. 3, 81 (2013) doi: 10.1186/2191-219X-3-81

[25] G. Nasso, R. Bonifazi, A. Del Prete, G. Del Prete, V. Lopriore, F. Bartolomucci, A. M. Calafiore and G. Speziale, J. Thorac. Cardiovasc. Surg. 142, e41 (2011) doi: 10.1016/j.jtcvs.2011.04.009 